\title{
Postcards from the EDge: 24-month outcomes of a randomised controlled trial for hospital-treated
}

\section{self-poisoning}

\author{
GREGORY L. CARTER, KERRIE CLOVER, IAN M. WHYTE, \\ ANDREW H. DAWSON and CATHERINE D'ESTE
}

\author{
Background Repetition of self- \\ poisoning is common.
}

\begin{abstract}
Aims To report the 24-month outcomes of a non-obligatory postcard intervention (plus treatment as usual) compared with treatment as usual.
\end{abstract}

\begin{abstract}
Method In a randomised-controlled trial (Zelen design) conducted in Newcastle, Australia, eight postcards were sent to participants over a 12-month period. The principal outcomes were the proportion of participants with one or more repeat episodes of self-poisoning and the number of repeat episodes per person.
\end{abstract}

Results No significant reduction was observed in the proportion of people repeating self-poisoning in the intervention group (21.2\%, 95\% Cl 17.025.3) compared with the control group (22.8\%, 95\% Cl 18.7-27.0; $\chi^{2}=0.32$, d.f. $=1$, $P=0.57)$; the difference between groups was $-1.7 \%(95 \% \mathrm{Cl}-7.5$ to 4.2$)$. There was a significant reduction in the rate of repetition, with an incidence risk ratio of 0.49 (95\% Cl 0.33-0.73).

\section{Conclusions A postcard intervention maintained the halving of the rate of repetition of hospital-treated self- poisoning events over a 2-year period, although it did not significantly reduce the proportion of individuals who repeated self-poisoning.}

Declaration of interest None.
Hospital-treated self-poisoning is common in Australia (McGrath, 1989). Self-harm is common in the UK (House et al, 1998), with self-poisoning being the most common form and with most patients making their initial contact with hospital through the emergency department (Gunnell et al, 2005). Repetition of self-harm within 12 months of an index episode is common, with a median rate of $16 \%$ (interquartile range $12-25 \%$ ), has a strong association with subsequent suicide and has significant resource implications for the health system (Owens et al, 2002). Five non-pharmacological interventions have been shown to be effective in reducing repetition of self-harm: one after 6 months' follow-up (Guthrie et al, 2001), three at the completion of the intervention duration of 12 months (Linehan et al, 1991; Brown et al, 2005; Carter et al, 2005); and one at treatment completion after 18 months (Bateman \& Fonagy, 1999). Only three studies have reported sustained, beneficial repetition outcomes beyond the intervention duration: one reported outcomes after 6 months (Guthrie et al, 2001), one after 36 months (18 months' follow-up; Bateman $\&$ Fonagy, 2001) and one after 24 months (12 months' follow-up; Linehan et al, 2006). We have previously shown that a postcard intervention reduced the rate of repetition of hospital-treated self-poisoning events over the 12-month period of the intervention (Carter et al, 2005). In this paper we report on the 24-month outcomes. There were two primary outcomes: the proportion of patients with at least one further episode; and the number of further admissions for self-poisoning per individual.

\section{METHOD}

The study methods have been previously reported in detail (Carter et al, 2005).

\section{Setting}

The Hunter Area Toxicology Service (HATS) is a regional toxicology unit at the Newcastle Mater Misericordiae Hospital, New South Wales, Australia, serving a primary referral population of 385000 adults and a tertiary referral population of a further 170000 . All poisoning presentations to emergency departments in the greater Newcastle region are either admitted to HATS or notified to HATS and entered prospectively into a clinical database (Whyte et al, 1997, 2002). The HATS model of service delivery means that in (virtually) all cases of selfpoisoning in the catchment area the person is brought to the Newcastle Mater Hospital for treatment and in all cases the person is formally admitted under the care of HATS. The psychiatry department sees all such patients for assessment and diagnosis, and to determine discharge destination and follow-up. Details of the model of service for these patients have been described by Whyte et al (1997).

\section{Study population}

Patients aged 16 years or over who presented to HATS during the recruitment period (April 1998 to December 2001) were potentially eligible. Inclusion criteria were that the patient must be capable of informed consent, not considered to pose a threat to an interviewer, not of 'no fixed address' and with sufficient English to complete a structured interview.

\section{Variables}

The two dependent variables were the proportion of individuals who had one or more readmissions for self-poisoning and the number of readmissions for self-poisoning per individual, over 24 months. Descriptive variables were obtained from the standardised clinical assessment of the patients (Buckley et al, 1999) and extracted from the HATS database. The categorical variables were gender, marital status (married, including de facto relationships, $v$. never married, separated, divorced or widowed), employment (full-time or part-time $v$. unemployed, pensioner, student or other), intensive care unit admission, time of admission (09.0017.00 weekdays $v$. out of hours) and discharge destination (psychiatric hospital $v$. all others). The continuous variables were age, length of stay in hours, median number of previous admissions to HATS for self-poisoning and number of psychiatric diagnoses from clinical assessment.

\section{Study design}

A randomised consent (Zelen: single consent version) design was used (Zelen, 
$1979,1990)$. This design is a variation on the standard randomised controlled experimental design, in which participants were randomised to control or intervention before consent was sought. In the single consent version, written informed consent to receive the intervention (eight nonobligatory postcards) was sought only from participants randomised to the intervention. The outcomes were assessed by an intention-to-treat analysis based on randomisation status.

The Hunter Area Health Research Ethics Committee approved this study, including the randomised consent design.

\section{Randomisation}

Randomisation was by database (HanDBase version 2.0; DDH Software, Wellington, Florida, USA) on a personal digital assistant (Palm III; Palm, Inc., Sunnyvale, California, USA) which was populated with a pre-generated randomisation schedule (in blocks of ten) and carried by the duty toxicologist. To avoid recruiting patients more than once, identification information was searched in this database before enrolment. To maintain masking to allocation status during recruitment, randomisation was not revealed until after all information was entered and eligibility determined. Randomisation status was then revealed in order to obtain patient consent. To monitor any potential alterations (interference) a duplicate record was kept in a hidden field of the database and a copy held on a separate computer for later verification of correct randomisation status.

All other clinical and research staff were unaware of allocation.

\section{Intervention}

A new intervention was developed based on the study by Jerome Motto, which demonstrated reduced death by suicide in a psychiatric hospital in-patient population (Motto, 1976; Motto \& Bostrom, 2001). The new intervention was a series of eight 'postcards' sent in a sealed envelope in months $1,2,3,4,6,8,10$ and 12 after discharge (the postcard is shown in the online supplement to this paper). All participants received treatment as usual.

\section{Sample size}

During study planning several sample sizes were calculated based on different estimates of possible effects for the 12-month outcomes
(Carter et al, 1999). A difference in proportions ( $5 \%$ significance level, $80 \%$ power) of $15 \%$ to $10 \%$ required 1364 participants, $20 \%$ to $10 \%$ required 392 participants and $30 \%$ to $20 \%$ required 293 participants. On the basis of clinical experience and previous research it was anticipated that $15-30 \%$ of the control group would self-poison again within 12 months, with an average of two episodes, meaning overall self-poisoning rates of $30-60 \%$. A sample of 400 per group would allow detection of absolute differences between groups of $10-15 \% \quad(5 \%$ significance level, 80\% power) and $12-17 \%$ (5\% significance level, $90 \%$ power), yielding relative risks of $0.67-0.75$ and $0.60-0.72(80 \%$ and $90 \%$ power respectively). This sample size would be adequate to detect differences in the proportion of participants who had any episode of self-poisoning of $7-9 \% \quad(80 \%$ power) and $8-10 \%$ (90\% power and $5 \%$ significance level), which we considered would represent a clinically significant reduction.

\section{Statistical analyses}

Data were analysed using the computerised statistical packages SPSS version 10.0 and Intercooled Stata versions 7 and 8 . The difference in proportions of participants with any readmission for self-poisoning was tested with $\chi^{2}$ analyses. For the number of readmissions per individual, a negative binomial regression was undertaken to compare the risk of self-poisoning events per individual in the postcard group relative to the control group and is reported as incidence risk ratio (IRR) with $95 \%$ confidence intervals. Two subgroup analyses, using negative binomial models, were undertaken for the treatment effect for male and female groups, since a post hoc analysis of 12month outcomes suggested a gender effect (Carter et al, 2005).

\section{Intent to treat}

We assessed 922 patients for eligibility, of whom 150 (16\%) were ineligible (Fig. 1), leaving 772 potential participants (control

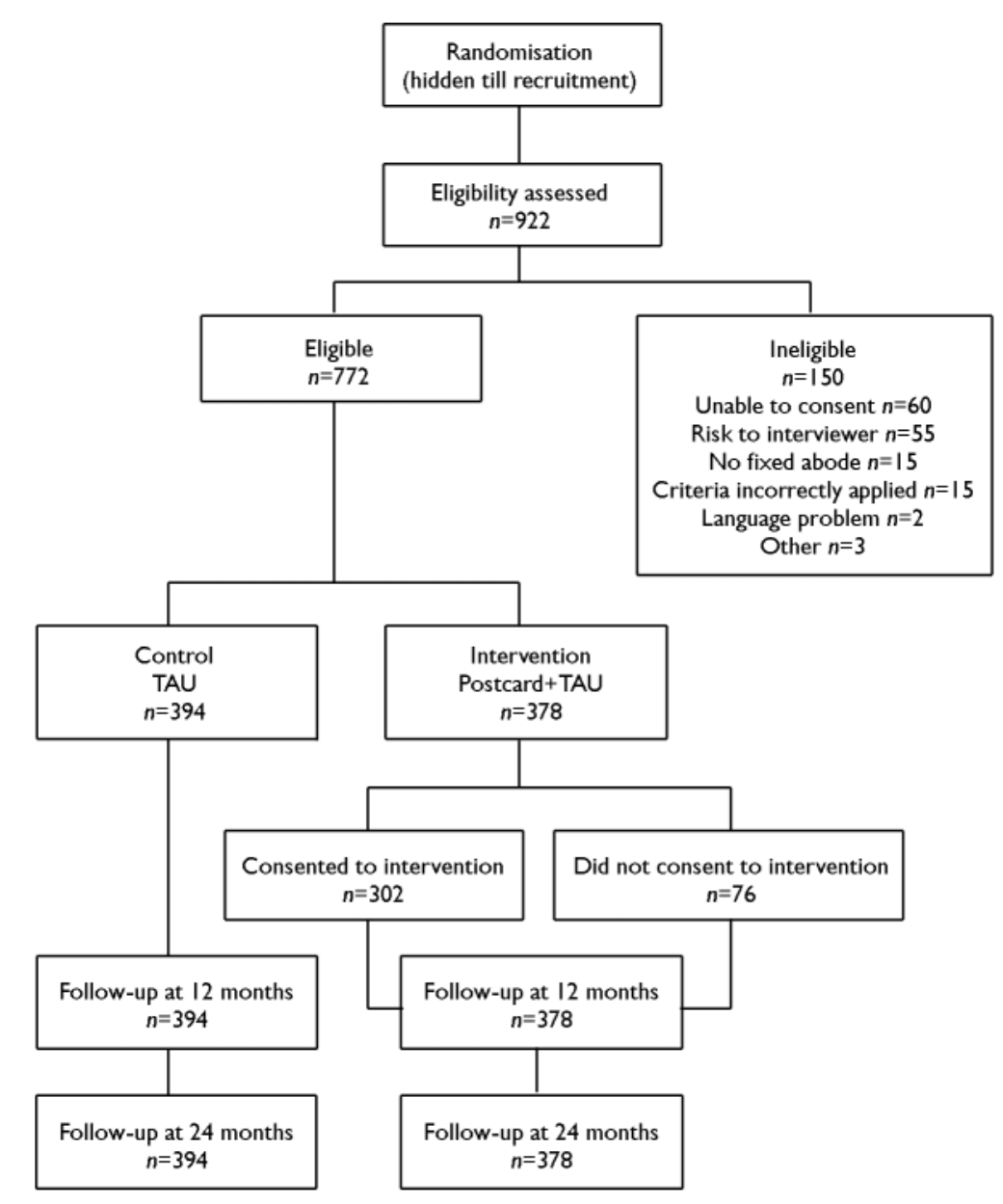

Fig. I Study profile (TAU, treatment as usual). 
group $n=394$ intervention group $n=378$ ). Among the intervention group, 76 refused the intervention, 1 missed the intervention as planned and 32 did not receive the full intervention (this was due to these people being unavailable, their postcards being returned 'not known at this address - return to sender'). Twenty people in the control group received the intervention owing to clerical errors, but were retained in the control group as data were analysed as intention to treat, based on randomisation.

\section{RESULTS}

The characteristics of the sample at baseline are summarised in Table 1. Previous episodes of hospital-treated self-poisoning were recorded for $17 \%$ of the total sample, (129/772), 17\% of the control group (66/ $394)$ and $17 \%$ of the intervention group (63/378). The frequency of the poisoning subtypes were: pharmaceuticals only, 473 (61\%); pharmaceuticals plus alcohol, 217 (28\%); opioid or amphetamine, $20(3 \%)$; carbon monoxide, $17(2 \%)$; herbicide or rodenticide, $11(1 \%)$; insulin, $7(1 \%)$; selfpoisoning with additional self-harm 23 (3\%); and unknown poison, $4(1 \%)$.

\section{Proportion of sample repeating self-poisoning}

In the intervention group 21.2\% (80/378; 95\% CI 17.0-25.3) had one or more readmissions for self-poisoning compared with $22.8 \%$ (90/394; 95\% CI 18.7-27.0) in the control group 24 months after baseline, a non-significant difference $\left(\chi^{2}=0.317\right.$, d.f. $=1, P=0.57)$, the difference between groups being $-1.7 \%$ (95\% CI -7.5 to 4.2$)$.

\section{Number of repeat admissions}

There were 310 cumulative readmissions in the control group and 145 in the intervention group (Fig. 2). Table 2 shows the relative risks for the intervention group compared with the control group from the negative binomial regressions. The risk of repetition was statistically significantly lower in the intervention group (IRR $=0.49$, $0.49,95 \%$ CI $0.33-0.73)$. Separate subgroup analyses by gender showed the treatment was effective for women $(\mathrm{IRR}=0.49$, 95\% CI $0.30-0.80)$ but not for men (IRR $=0.97,95 \%$ CI 0.50-1.88. Readmissions by intervention group and gender are shown in Table 3.
Table I Characteristics of the study sample

\begin{tabular}{|c|c|c|c|c|}
\hline & $\begin{array}{c}\text { Missing } \\
\text { data } \\
n\end{array}$ & $\begin{array}{c}\text { Total } \\
\text { sample } \\
(n=772)\end{array}$ & $\begin{array}{l}\text { Control } \\
\text { group } \\
(n=394)\end{array}$ & $\begin{array}{l}\text { Intervention } \\
\text { group } \\
(n=378)\end{array}$ \\
\hline \multicolumn{5}{|l|}{ Categorical variables, $n$ (\%) } \\
\hline Female & 1 & $524(68)$ & $291(74)$ & $233(62)$ \\
\hline Married' & 31 & $256(35)$ & $118(31)$ & $138(38)$ \\
\hline Employed & 153 & $162(26)$ & $88(27)$ & $74(26)$ \\
\hline Admitted to intensive care & 0 & $113(15)$ & $60(15)$ & $53(14)$ \\
\hline Admitted outside office hours & 0 & $581(75)$ & $296(75)$ & $285(75)$ \\
\hline Discharged to psychiatric hospital & 2 & $208(27)$ & $106(27)$ & $102(27)$ \\
\hline Previous admission for self-poisoning & 0 & $129(17)$ & $66(17)$ & $63(17)$ \\
\hline \multicolumn{5}{|l|}{ Continuous variables, median $(\mathrm{Q} \mathrm{I}-\mathrm{Q} 3)^{2}$} \\
\hline Age, years & 0 & $33(24-44)$ & $34(23-45)$ & $33(24-42)$ \\
\hline Length of stay, $\mathrm{h}$ & 0 & $18(12-30)$ & $18(|3-3|)$ & $17(12-29)$ \\
\hline Number of prior self-poisoning admissions & 0 & 0 & 0 & 0 \\
\hline Number of psychiatric diagnoses & 0 & $2(I-2)$ & $2(I-3)$ & $2(I-3)$ \\
\hline
\end{tabular}

I. Includes de facto relationships.

2. First quartile-third quartile.

\section{DISCUSSION}

\section{Strengths and weaknesses of the study}

This study had several strengths. First, a randomised consent design was used, which was suited to this study and this clinical population. In this study the baseline characteristics and principal outcomes were tracked by the HATS database, allowing for complete follow-up. Second, the quality of the randomisation was strong, with randomisation undertaken using a handheld personal computer device in combination with another computer which could detect any errors of allocation status and interference with the randomisation. Third, all participants, clinicians and research staff were masked to the primary outcome measurement; only the recruiting toxicologists and the secretary responsible for managing the mailing database and postcards were aware of allocation status. Owing to the randomised design, the quality of masking to allocation and to outcome, and the service model of HATS in which all cases of self-poisoning are admitted to hospital, we believe that there are few threats to internal validity in this study.

There were some limitations to consider for this study and caution needs to be used when interpreting the results. Less than a quarter of the participants self-poisoned a second time, and a subgroup showed a highly skewed pattern of more than one repeat episode. It is not known to what extent the HATS referral population and model of clinical service (Whyte et al, 1997) would be generalisable to other settings. This study investigated patients who self-poisoned and the results cannot necessarily be generalised to patients with other forms of self-harm. We do not currently have data available on mortality and suicide outcomes for the study participants. A previous longitudinal study in our centre found a $1 \%$ suicide rate after 24 months and nearly $2 \%$ suicide rate after 5 years, which would translate to 8 suicide deaths and 16 suicide deaths respectively (Reith et al, 2004). These suicide rates are lower than the 12-month $1.8 \%$ rate reported in a recent meta-analysis of psychosocial interventions after self-harm (Crawford et al, 2007). We intend to be able to report the mortality and suicide outcomes for the 5-year follow-up of the Postcards from the EDge project in the future.

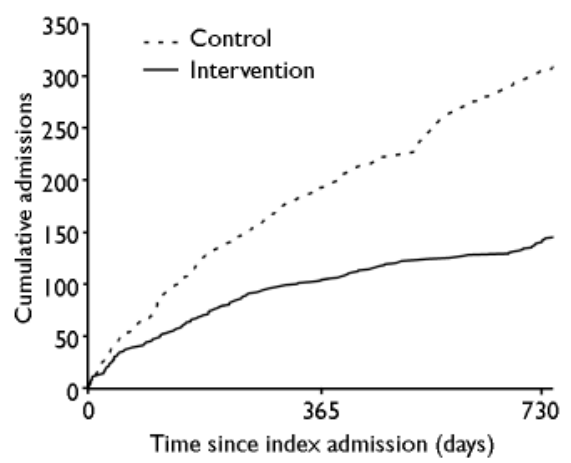

Fig. 2 Cumulative readmissions to hospital for treatment of self-poisoning (24 months; unadjusted for multiple admissions). 
Table 2 Negative binomial models for 24-month outcomes for intervention effect on number of readmissions for self-poisoning and for subgroup analyses by gender

\begin{tabular}{|c|c|c|c|c|c|}
\hline & \multicolumn{3}{|c|}{ Incidence risk ratio } & \multicolumn{2}{|c|}{ Wald test } \\
\hline & IRR & s.e. & $95 \% \mathrm{Cl}$ & $Z$ & $P$ \\
\hline \multicolumn{6}{|c|}{ Whole sample, unadjusted $(n=772)$} \\
\hline Control & 1.00 & & & & \\
\hline Intervention & 0.49 & 0.10 & $0.33-0.73$ & -3.51 & 0.010 \\
\hline \multicolumn{6}{|l|}{ Men $(n=247)$} \\
\hline Control' & 1.00 & & & & \\
\hline Intervention & 0.97 & 0.33 & $0.50-1.88$ & -0.09 & 0.929 \\
\hline \multicolumn{6}{|l|}{ Women $(n=524)$} \\
\hline Control' & 1.00 & & & & \\
\hline Intervention & 0.49 & 0.12 & $0.30-0.80$ & -2.87 & 0.004 \\
\hline
\end{tabular}

I. One control group participant with missing data for gender (13 readmissions) is not included in either gender subgroup analysis.

Replications of this study and additional effectiveness trials would be necessary before widespread implementation could be considered. The decision to include a subgroup analysis based on gender was a post hoc one based on the findings from the primary outcomes at 12 months. Caution should always be used in interpreting such subgroup analyses because of (unplanned) reduced sample sizes, and judgement needs to be exercised regarding the biological plausibility of such analyses. There may also have been a conservative estimate of treatment effect because 20 persons in the control group were inadvertently exposed to the intervention, whereas 76 persons in the treatment group did not consent to receive the intervention and a further 32 persons were not exposed to the intervention.

\section{Implications of the study}

The simple postcard intervention used in this study was previously shown to have nearly halved the number of readmissions for self-poisoning within 12 months: $\mathrm{IRR}=0.55,95 \%$ CI $(0.35-0.87)$ (Carter et al, 2005). This study of 24-month outcomes showed that this benefit was maintained for a further 12 months after the final contact by postcard: $I R R=0.49,95 \%$ CI $0.33-0.73$. There were 422 bed-days used by the control group and 183 bed-days used by the postcard group, a total of 239 bed-days saved. This represented a considerable ongoing saving in opportunity costs, availability of hospital beds and decrease in emergency department workload. This lowcost intervention seems to have substantial cost-effectiveness. The simplicity of the intervention means that it could be delivered resources. The difference in total readmissions for self-poisoning came from one main source - women with three or more accounted for a difference of 165 repeat admissions (210 in the control group and 45 in the intervention group). from hospitals that do not have extensive repeat admissions (see Table 3) - which

\section{Benefit of treatment beyond the treatment phase}

One other study ( $n=101)$ has shown a significant reduction in self-harm over a 24month period (12 months after treatment cessation): $23 \% \quad v .46 \%$ (Linehan et al, 2006). That study used dialectical behaviour therapy to treat women with recent self-harming behaviour who met criteria for borderline personality disorder. This therapy is an important form of treatment for a subset of patients who self-harm, although it is not applicable universally to the hospital-treated population. One further study $(n=44)$ has shown a sustained reduction in self-mutilation $(23 \% v .68 \%)$ and suicide gesture $(18 \% v .63 \%)$ over 36 months, 18 months after treatment cessation (Bateman \& Fonagy, 2001). That study used a psychoanalytically informed day hospital programme in patients with borderline personality disorder. Both of these studies suggested that longer-term alternative behaviours to self-harm were learned, such as improvement in coping strategies, emotion regulation, impulse control, self-understanding or relationship quality, which resulted in the maintenance of the beneficial effects (Bateman \& Fonagy, 2001; Linehan et al, 2006). A third study of self-poisoning patients $(n=119)$, using a brief, nurse-led psychological intervention in the patient's home, found the intervention group less likely to report repeated attempts to harm themselves at the 6-month follow-up (9\% v. 28\%; Guthrie et al, 2001). Beneficial effects on repetition sustained beyond the duration of the intervention might have been mediated by other beneficial effects on suicidal ideation and treatment satisfaction. All three of these studies used highly selected subgroups within the hospitaltreated self-harm population, either people

Table 3 Number of repetitions of self-poisoning by gender and experimental group

\begin{tabular}{|c|c|c|c|c|c|c|c|c|}
\hline \multirow{3}{*}{$\begin{array}{l}\text { Number of } \\
\text { repetitions }\end{array}$} & \multicolumn{4}{|c|}{ Control group $(n=394)^{\prime}$} & \multicolumn{4}{|c|}{ Intervention group $(n=378)$} \\
\hline & \multicolumn{2}{|c|}{ Men $(n=102)$} & \multicolumn{2}{|c|}{ Women $(n=291)$} & \multicolumn{2}{|c|}{ Men $(n=145)$} & \multicolumn{2}{|c|}{ Women $(n=233)$} \\
\hline & $n(\%)$ & Total repetitions & $n(\%)$ & Total repetitions & $n(\%)$ & Total repetitions & $n(\%)$ & Total repetitions \\
\hline None & $83(8 I)$ & 0 & $221(76)$ & 0 & $119(82)$ & 0 & $179(77)$ & 0 \\
\hline One & $14(14)$ & 14 & $34(12)$ & 34 & $19(13)$ & 19 & $32(14)$ & 32 \\
\hline Two & $3(3)$ & 6 & $12(4)$ & 24 & $4(3)$ & 8 & $14(6)$ & 28 \\
\hline Three or more & $2(2)$ & 9 & $24(8)$ & 210 & $3(2)$ & 13 & $8(3)$ & 45 \\
\hline
\end{tabular}

I. One control group participant with missing data for gender had 13 readmissions. 
with borderline personality disorder (Bateman \& Fonagy, 2001; Linehan et al, 2006) or a group of only 119 participants from an initial sample of 587 patients presenting with self-poisoning (Guthrie et al, 2001), which makes comparison with our study more difficult. However, these four studies taken together suggest that if some impact on reduction of repetition of selfharm or self-poisoning behaviour can be made, then perhaps the effects might be sustained over a period beyond that of the intervention.

Although it may also be inferred that some participants in our study learned sustained alternative behaviours to selfpoisoning, there was no particular component of the intervention that aimed to induce these specific behavioural changes. The mechanism for the long-term benefits in the Postcards from the EDge study remains speculative. We have planned a qualitative study to explore the question of what mechanisms might have contributed to this. We also do not know whether there was any change in the pattern of psychiatric hospital, psychiatric community service or primary care service use that might have been a mediating factor in the difference in repetition events, but we hope to be able to explore some of these possibilities in the planned 5-year follow-up study.

\section{Comparison with other brief interventions}

There are other brief interventions for repetition of hospital-treated self-harm or selfpoisoning that can be considered. One of the best-known low-cost interventions for self-harm in the UK was the green card study and the subsequent crisis telephone card study, which showed no difference in the proportion of those repeating self-harm at 6 months (Evans et al, 1999) or at 12 months (Morgan et al, 1993; Evans et al, $2005)$. The first of these studies $(n=212)$ was underpowered and included only those presenting for the first time with self-harm, who subsequently showed a repeat rate of self-harm of only $8 \%$ (Morgan et al, $1993)$. The second variation $(n=827)$ was adequately powered, used a self-harm case register to define repetition, and found no difference in proportions of those repeating self-harm, with a 12-month repetition rate of $20 \%$ (Evans et al, 2005). In France, a telephone contact intervention delivered either 1 month or 3 months after an episode of self-poisoning showed no difference in the proportion of a combined 'adverse effects' outcome or proportion of selfreported suicide attempt (Vaiva et al, 2006). That study ( $n=605)$ was adequately powered, used a stratified (based on more than four suicide attempts in 3 years), three-arm design and had a 12-month repeat attempted suicide rate of $17 \%$. A multicentre UK study used a manualassisted cognitive-behavioural therapy intervention for established cases of repeated self-harm and showed no difference in proportion of those with self-reported repeat parasuicide (Tyrer et al, 2003). This study $(n=480)$ was adequately powered (for an expected repetition rate of $45 \%$ ), used a stratified (based on parasuicide score) design and had a 12-month parasuicide rate of $43 \%$. The comparison condition for these studies was standard treatment or treatment as usual and for the Postcards from the EDge study it was postcards plus treatment as usual $v$. usual treatment alone. Each of these studies showed a similar non-significant reduction in the proportion of those repeating selfharm (patient rate) of 2.2-7.0\% (Crawford $\&$ Kumar, 2007). However, none of these studies of brief, low-cost interventions analysed the number of episodes of repeated self-harm (event rates) as an outcome, and most used different definitions of the primary outcome and different methods of estimating the rates of that outcome, so comparison with the beneficial finding from the Postcards from the EDge study is difficult despite the similarities of intent underlying the interventions.

\section{Concluding remarks}

This study of hospital-treated self-poisoning showed that a low-cost postcard intervention was effective in reducing the number of events per individual by a relative reduction of more than $50 \%$, which was clinically and statistically significant. The postcard intervention continued to be effective in reducing repeat episodes of hospital-treated self-poisoning for 1 year after the intervention ceased.

\section{ACKNOWLEDGEMENTS}

We thank Dr Jerome Motto for his encouragement to use and modify the original intervention for this project. We gratefully acknowledge the contribution of Dr Trish McGettigan, Dr Patrick Oakley, Dr Nav Gupta and Dr Geoff Isbister for patient recruitment and Mr Stuart Allen for data management support. We would also like to thank Professor Bob Goldney and Dr Natalia Carter for alerting us to the Motto study, Mrs Debra Whyte and Mrs Antonia Nash for data entry and Dr Julia Lowe and Dr Dianne O'Connell for schooling us in Zelen designs. Finally, we thank Mrs Helen Rowsell, the departmental secretary, who managed the mailing database and mailing lists so diligently over a long period.

\section{REFERENCES}

Bateman, A. \& Fonagy, P. (1999) Effectiveness of partial hospitalization in the treatment of borderline personality disorder: a randomized controlled trial. American Journal of Psychiatry, 156, 1563-1569.

Bateman, A. \& Fonagy, P. (200I) Treatment of borderline personality disorder with psychoanalytically oriented partial hospitalization: an 18-month follow-up. American Journal of Psychiatry, 158, 36-42.

Brown, G. K., Ten Have, T., Henriques, G. R., et al (2005) Cognitive therapy for the prevention of suicide attempts: a randomized controlled trial. JAMA, 294, 563-570.

Buckley, N. A., Whyte, I. M., Dawson, A. H., et al (1999) Preformatted admission charts for poisoning admissions facilitate clinical assessment and research. Annals of Emergency Medicine, 34, 476-482.

Carter, G., Clover, K. \& Fryer, J. (1999) Deliberate self-harm: can we move the goal posts closer? eBMJ, http://www.bmj.com/cgi/eletters/3I7/7I56/44

Carter, G. L., Clover, K., Whyte, I. M., et al (2005)

Postcards from the EDge project: randomised controlled trial of an intervention using postcards to reduce repetition of hospital treated deliberate self poisoning. BMJ, 33I, 805-807.

Crawford, M. J. \& Kumar, P. (2007) Intervention following deliberate self-harm: enough evidence to act? Evidence-Based Mental Health, 10, 37-39.

Crawford, M. J., Thomas, O., Khan, N., et al (2007) Psychosocial interventions following self-harm: systematic review of their efficacy in preventing suicide. British Journal of Psychiatry, 190, 11-17.

Evans, M. O., Morgan, H. G., Hayward, A., et al (1999) Crisis telephone consultation for deliberate selfharm patients: effects on repetition. British Journal of Psychiatry, 175, 23-27.

Evans, J., Evans, M., Morgan, H. G., et al (2005) Crisis card following self-harm: 12-month follow-up of a randomised controlled trial. British Journal of Psychiatry, 187, 186-187.

Gunnell, D., Bennewith, O., Peters, T. J., et al (2005)

The epidemiology and management of self-harm amongst adults in England. Journal of Public Health, 27, 67-73.

Guthrie, E., Kapur, N., Mackway-Jones, K., et al (200I) Randomised controlled trial of brief psychological intervention after deliberate self poisoning. $B M J, \mathbf{3 2 3}$, 135-138.

House, A., Owens, D. \& Patchett, L. (1998) Deliberate self-harm. Effective Health Care, 4, I-12.

Linehan, M. M., Armstrong, H. E., Suarez, A., et a (199I) Cognitive-behavioral treatment of chronically parasuicidal borderline patients. Archives of General Psychiatry, 48, 1060-1064.

Linehan, M. M., Comtois, K. A., Murray, A. M., et a (2006) Two-year randomized controlled trial and follow-up of dialectical behavior therapy vs therapy by experts for suicidal behaviors and borderline personality disorder. Archives of General Psychiatry, 63, 757-766 
McGrath, J. (1989) A survey of deliberate selfpoisoning. Medical Journal of Australia, I50, 317-322.

\section{Morgan, H. G., Jones, E. M. \& Owen, J. H. (1993)} Secondary prevention of non-fatal deliberate self-harm The green card study. British Journal of Psychiatry, $\mathbf{1 6 3}$, III-II2.

Motto, J. A. (1976) Suicide prevention for high-risk persons who refuse treatment. Suicide and LifeThreatening Behavior, 6, 223-230.

Motto, J. A. \& Bostrom, A. G. (200I) A randomized controlled trial of postcrisis suicide prevention. Psychiatric Services, 52, 828-833.

Owens, D., Horrocks, J. \& House,A. (2002) Fatal and non-fatal repetition of self-harm: systematic review. British Journal of Psychiatry, 18I, 193-199.

Reith, D. M., Whyte, I. M., Carter, G. L., et al (2004) Risk factors for suicide and other deaths following hospital treated self-poisoning in Australia. Australian and New Zealand Journal of Psychiatry, 38, 520-525.

Tyrer, P., Thompson, S., Schmidt, U., et al (2003) Randomized controlled trial of brief cognitive behaviour therapy versus treatment as usual in recurrent deliberate self-harm: the POPMACT study. Psychological Medicine, 33, 969-976.

Vaiva, G., Ducrocq, F., Meyer, P., et al (2006) Effect of telephone contact on further suicide attempts in patients

GREGORY L. CARTER, Suicide Prevention Research Unit, Centre for Mental Health Studies, Faculty of Health, University of Newcastle, New South Wales, and Department of Consultation-Liaison Psychiatry, Newcastle Mater Hospital, Newcastle; KERRIE CLOVER, Suicide Prevention Research Unit, Centre for Mental Health Studies, and Faculty of Health, University of Newcastle; IAN M.WHYTE, Discipline of Clinical Pharmacology, Faculty of Health, University of Newcastle, and Department of Clinical Toxicology and Pharmacology, Newcastle Mater Hospital, Newcastle; ANDREW H. DAWSON, Discipline of Clinical Pharmacology, Faculty of Health, University of Newcastle, Australia, and South Asian Clinical Toxicology Research Collaboration University of Peradeniya, Sri Lanka; CATHERINE D'ESTE, Centre for Clinical Epidemiology and Biostatistics, Faculty of Health, University of Newcastle, and Centre for Military and Veterans' Health, University of Queensland, Brisbane, Australia

Correspondence: Dr Gregory Carter, Department of Consultation - Liaison Psychiatry, Locked Bag 7, Hunter Region Mail Centre, NSW 2310, Australia. Tel: + 61 24921 1283; fax: + 61 24921 1870;

email: Gregory.Carter@newcastle.edu.au

(First received 28 March 2007, final revision 5 June 2007, accepted 17 July 2007)

discharged from an emergency department: randomised controlled study. BMJ, 332, 124I-1245.

Whyte, I. M., Dawson, A. H., Buckley, N. A., et al (1997) A model for the management of self-poisoning. Medical Journal of Australia, 167, 142-146.

Whyte, I. M., Buckley, N. A. \& Dawson, A. H. (2002) Data collection in clinical toxicology: are there too many variables? Journal of Toxicology - Clinical Toxicology, $\mathbf{4 0}$, 223-230.

Zelen, M. (1979) A new design for randomized clinical trials. New England Journal of Medicine, 300, 1242-1245.

Zelen, M. (1990) Randomized consent designs for clinical trials: an update. Statistics in Medicine, 9, 645656. 